\title{
3-day mortality in hospitalised community-acquired pneumonia: frequency and risk factors
}

\author{
To the Editor:
}

Mortality of hospitalised patients with community-acquired pneumonia (CAP) continues to be high and efforts have been made to characterise a subgroup of patients with an emergency presentation defined by a high risk of early clinical deterioration or death in order to target initial management interventions to patients with a high potential of prognosis improvement [1-3]. However, the actual magnitude of an emergency presentation in CAP remains unclear as the most severely affected patients are frequently underrepresented in prospective studies [1,3]. Additionally, early mortality within 3 days in CAP only rarely has been studied so far [3-5]. The aim of the present analysis was to characterise patients dying within 3 days after hospitalisation for CAP using the unique database of the German nationwide mandatory quality assurance programme, which requests all hospitals in Germany to document all cases hospitalised with CAP according to a pre-specified electronic data sheet.

For this study, data from the 5-year period between 2009 and 2013 were included. Cases with CAP were identified by a set of International Classification of Diseases codes as listed elsewhere [1]. Comorbidity was not included in the analysis since it is not consistently documented in all patients and because there are no data on its severity. In addition, there are no data on Do Not Resuscitate orders or causes of death. The primary outcome parameter was death within 3 days after hospital admission. The CRB-65 (confusion, respiratory rate $\geqslant 30$ breaths per $\mathrm{min}$, systolic blood pressure $<90 \mathrm{mmHg}$ or diastolic blood pressure $\leqslant 60 \mathrm{mmHg}$ ) and age $\geqslant 65$ years) score was determined as described previously [6]. After 2010, respiratory rate was not a mandatory field to be recorded; cases with missing respiratory rate were considered normal. For univariate analyses, continuous variables were compared using Student's t-test and categorical variables using the Chi-squared test. To evaluate the independent association of predictive parameters with 3-day mortality, a multivariate logistic regression model with stepwise forward selection was performed. Risk factors included were the CRB-65 criteria and mechanical ventilation on admission. Receiver operating characteristic (ROC) curve analysis was used to determine the diagnostic properties of the CRB-65 score.

A total of 1195461 patients with hospitalised CAP were documented in Germany between 2009 and 2013. Of those, 49538 patients transferred from other hospitals were excluded in order to avoid redundant documentation. The remaining 1145923 patients showed a hospital mortality of $13.1 \%$ (150116 out of 1145923 ). Of these deaths, 38.6\% (58087) occurred within the first 3 days of hospitalisation (3-day mortality $5.1 \%)$.

In order to overcome the problem of treatment restrictions in patients already severely compromised before the CAP episode, patients who were either bedridden prior to admission and/or residing in a nursing home, and/or not receiving antibiotic treatment (366207 cases; 3-day mortality $10.8 \%$, hospital mortality $25.7 \%$ ) were excluded from the analysis. The remaining 779716 patients showed a 3-day mortality of $2.4 \%$ (18474 deaths), corresponding to $33 \%$ of all in-hospital deaths (56250; hospital mortality 7.2\%). Characteristics of these patients are shown in table 1 . Age, sex and the CRB-65 criteria were significantly associated with 3-day mortality. In patients with mechanical ventilation, the 3 -day mortality rate was $8.6 \%$, as compared to $1.9 \%$ in patients without mechanical ventilation $(\mathrm{p}<0.001)$. However, of the patients dying within 3 days, only $23.3 \%$ had received mechanical ventilation before. In multivariate analysis, mechanical ventilation on admission showed the highest risk of 3-day mortality. Additionally, all CRB-65 criteria were independently associated with 3-day mortality, with pneumonia-related confusion showing the highest risk. 3-day mortality increased with each CRB-65 point and was 0.6\% in CRB-65 0, 1.9\% in CRB-65 1, 4.2\% in CRB-65 2, 10.4\% in CRB-65 3 and 20.9\% in CRB-65 4. After ROC analysis, the CRB-65 score showed an area under the curve (AUC) of 0.69 (95\% CI $0.68-0.69, \mathrm{p}<0.001)$ for predicting 3-day mortality. Optimal cut-off according to the Youden index was two or more criteria (sensitivity $51.3 \%$, specificity $77.7 \%$ ). A score of 0 showed a negative predictive value of $99.4 \%$ (negative likelihood ratio 0.27 ), whereas scores of three or more and four criteria had positive predictive values of $11.4 \%$ (positive likelihood ratio 5.25) and 20.9\% (positive likelihood ratio 10.0), respectively.

The main strength of our findings lies in the evaluated database virtually excluding any selection bias by including all German hospitalised CAP patients, thus allowing an unparalleled, scientifically and educationally important insight into epidemiology and the often underestimated emergency presentation of CAP. Our data demonstrate that there is a relevant subgroup of CAP patients dying within 3 days of hospital admission, even after exclusion of patients with poor functional status, nursing home residents and those not receiving 
TABLE 1 Characteristics of patients and multivariate regression analysis including CRB-65 criteria and mechanical ventilation on admission according to 3-day mortality

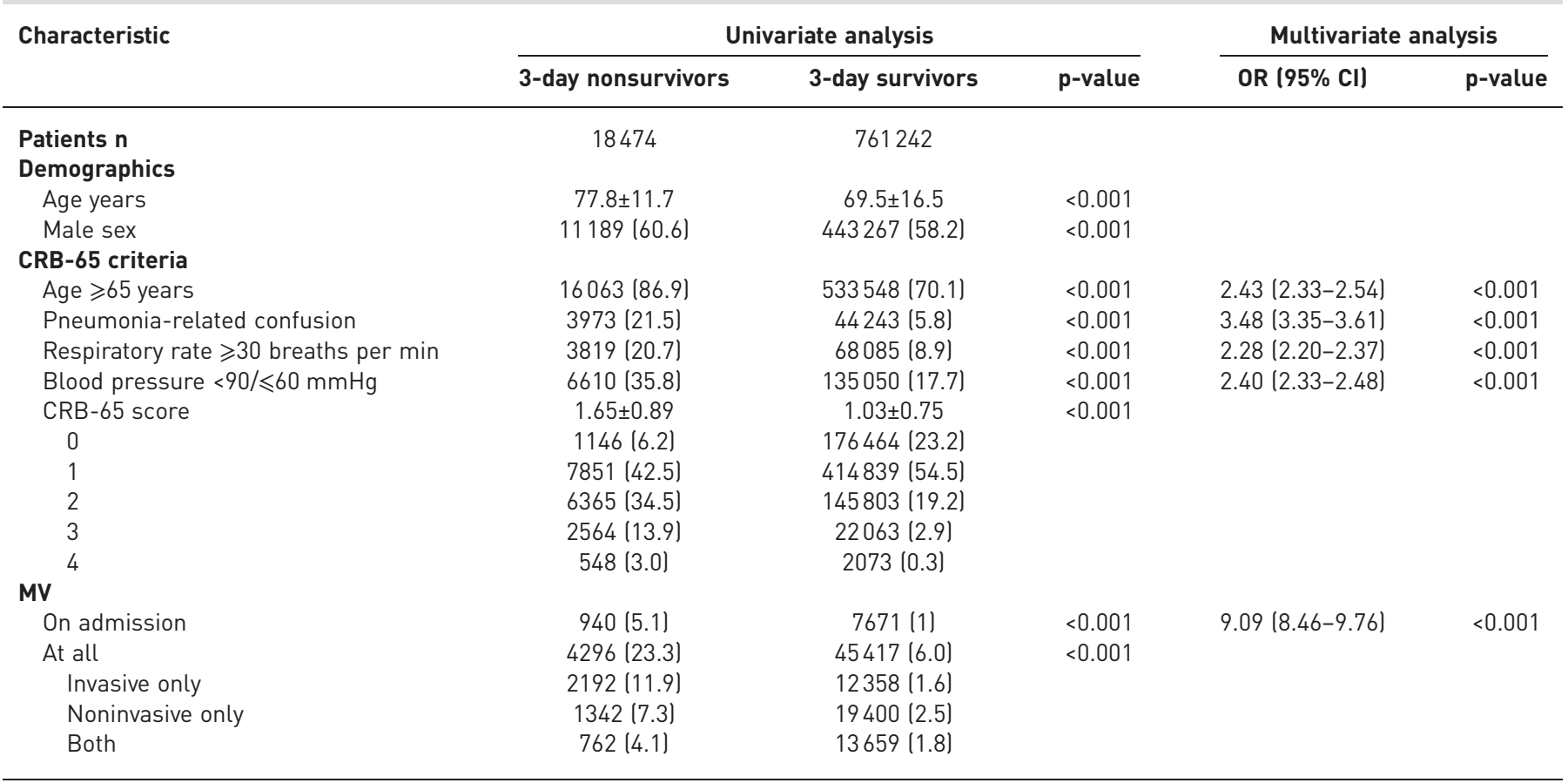

Data are presented as mean \pm SD or $n(\%)$, unless otherwise stated. MV: mechanical ventilation.

antibiotic treatment to avoid bias due to possible treatment restrictions. The resulting 3-day mortality rate of $2.4 \%$ is higher than that reported by two recent populations from prospective studies evaluating this end-point $[3,4]$ and still high when compared to the reported 48 -h mortality rate of $2.3 \%$ in a study including all patients with CAP in a Spanish hospital without excluding nursing home or bedridden patients [5]. For Germany, this means that nearly 3700 of these patients per year are affected. This has implications for planning and evaluating possible interventions in order to improve management of these patients.

Our mechanical ventilation rate before death on day 3 of only $23.3 \%$, even after exclusion of patients who were bedridden or residing in a nursing home, matches that of a recent analysis of this database and could reflect either treatment restrictions or deficient clinical performance [7].

As far as we know, no other study evaluated the CRB-65 score for 3-day mortality so far. Our data demonstrate that the negative predictive value of a CRB-65 of 0 was very high (99.4\%). However, even this favourable performance implies that 1146 patients not being bedridden or coming from a nursing home with a score of 0 died within 3 days in Germany during the 5-year study period. Additionally, the AUC of 0.69 for prediction of 3-day mortality in our database was lower than that reported by recent studies and meta-analyses for hospital mortality $[8,9]$, which might reflect a poorer performance of this score for this early outcome. Strategies that may reduce the risk of early death despite a low CRB-65 score include evaluation of sepsis- or comorbidity-related organ dysfunction, including oxygenation and other proposed parameters like the American Thoracic Society/Infectious Diseases Society of America (ATS/IDSA) minor criteria [10-13]. Moreover, patients should be clinically assessed repeatedly during the first 3 days of hospitalisation [14, 15].

Several limitations of our study have to be mentioned. The interpretation of our findings is limited by the lack of data on other important risk factors and management parameters like comorbidities, microbiology, intensive care unit admission, appropriateness of antimicrobial therapy, course of deterioration of patients and treatment restrictions, as valid information on these parameters cannot be retrieved from the quality performance programme. In addition, we cannot provide data on causes of death. Finally, other proposed risk scores like the Pneumonia Severity Index or the ATS/IDSA minor criteria are not available from the database. However, the coverage of virtually all hospitalised CAP patients in Germany reflects real-world management and minimises selection bias. Moreover, data completeness of the predefined outcome parameters as well as the CRB-65 score makes the evaluated database unique within the published CAP research.

In conclusion, we found a high 3-day mortality rate of $2.4 \%$ (one-third of all in-hospital deaths) in a large cohort of hospitalised CAP patients who were not bedridden or residing in nursing homes. The CRB-65 
score showed a high negative predictive value for early death. Patients presenting with severity criteria should be carefully evaluated for sepsis- or comorbidity-related acute organ dysfunction. independent predictors http://ow.ly/XFMlB

Martin Kolditz ${ }^{1}$, Torsten T. Bauer ${ }^{2}$, Thomas König ${ }^{3}$, Gernot Rohde ${ }^{4,5,6}$ and Santiago Ewig ${ }^{7}$

${ }^{1}$ Division of Pulmonology, Medical Dept I, University Hospital Carl Gustav Carus, Technische Universität Dresden, Dresden, Germany. ${ }^{2}$ HELIOS Klinikum Emil von Behring, Lungenklinik Heckeshorn, Berlin, Germany. ${ }^{3}$ Aqua-Institut, Göttingen, Germany. ${ }^{4}$ Dept of Respiratory Medicine, Maastricht University Medical Center, Maastricht, The Netherlands. ${ }^{5}$ CAPNETZ Stiftung, Hannover, Germany. ${ }^{6}$ Biomedical Research in Endstage and Obstructive Lung Disease Hannover (BREATH), Member of the German Center for Lung Research (DZL), Hannover, Germany. ${ }^{7}$ Thoraxzentrum Ruhrgebiet, Dept of Respiratory and Infectious Diseases, EVK Herne and Augusta-Kranken-Anstalt Bochum, Bochum, Germany.

Correspondence: Martin Kolditz, Division of Pulmonology, Medical Dept I, University Hospital Carl Gustav Carus, Fetscherstr.74,01307 Dresden, Germany. E-mail: martin.kolditz@uniklinikum-dresden.de

Received: Nov 302015 | Accepted after revision: Jan 192016 | First published online: Feb 252016

Conflict of interest: Disclosures can be found alongside the online version of this article at erj.ersjournals.com

Acknowledgements: The authors thank all physicians and technicians working for and with the quality control initiative for community acquired pneumonia and the AQUA Institute, Gottingen, Germany.

\section{References}

1 Ewig S, Birkner N, Strauss R, et al. New perspectives on community-acquired pneumonia in 388406 patients. Results from a nationwide mandatory performance measurement programme in healthcare quality. Thorax 2009; 64: 1062-1069.

2 Ewig S, Woodhead M, Torres A. Towards a sensible comprehension of severe community-acquired pneumonia. Intensive Care Med 2011; 37: 214-223.

3 Kolditz M, Ewig S, Klapdor B, et al. Community-acquired pneumonia as medical emergency: predictors of early deterioration. Thorax 2015; 70: 551-558.

4 Renaud B, Schuetz P, Claessens YE, et al. Proadrenomedullin improves risk of early admission to ICU score for predicting early severe community-acquired pneumonia. Chest 2012; 142: 1447-1454.

5 Garcia-Vidal C, Fernandez-Sabe N, Carratala J, et al. Early mortality in patients with community-acquired pneumonia: causes and risk factors. Eur Respir J 2008; 32: 733-739.

6 Lim WS, van der Eerden MM, Laing R, et al. Defining community acquired pneumonia severity on presentation to hospital: an international derivation and validation study. Thorax 2003; 58: 377-382.

7 Bauer TT, Welte T, Strauss R, et al. Why do nonsurvivors from community-acquired pneumonia not receive ventilatory support? Lung 2013; 191: 417-424.

8 Chalmers JD, Singanayagam A, Akram AR, et al. Severity assessment tools for predicting mortality in hospitalised patients with community-acquired pneumonia. Systematic review and meta-analysis. Thorax 2010; 65: 878-883.

9 Loke YK, Kwok CS, Niruban A, et al. Value of severity scales in predicting mortality from community-acquired pneumonia: systematic review and meta-analysis. Thorax 2010; 65: 884-890.

10 Kolditz M, Ewig S, Schutte H, et al. Assessment of oxygenation and comorbidities improves outcome prediction in patients with community-acquired pneumonia with a low CRB-65 score. J Intern Med 2015; 278: 193-202.

11 Aliberti S, Brambilla AM, Chalmers JD, et al. Phenotyping community-acquired pneumonia according to the presence of acute respiratory failure and severe sepsis. Respir Res 2014; 15: 27.

12 Chalmers JD, Taylor JK, Mandal P, et al. Validation of the Infectious Diseases Society of America/American Thoratic Society minor criteria for intensive care unit admission in community-acquired pneumonia patients without major criteria or contraindications to intensive care unit care. Clin Infect Dis 2011; 53: 503-511.

13 Chalmers JD, Mandal P, Singanayagam A, et al. Severity assessment tools to guide ICU admission in community-acquired pneumonia: systematic review and meta-analysis. Intensive Care Med 2011; 37: 1409-1420.

14 Dremsizov T, Clermont G, Kellum JA, et al. Severe sepsis in community-acquired pneumonia: when does it happen, and do systemic inflammatory response syndrome criteria help predict course? Chest 2006; 129: 968-978.

15 Phua J, Ngerng WJ, Lim TK. The impact of a delay in intensive care unit admission for community-acquired pneumonia. Eur Respir J 2010; 36: 826-833. 